\title{
VIMOS-VLT integral field kinematics of the giant low surface brightness galaxy ESO 323-G064^
}

\author{
L. Coccato ${ }^{1}$, R. A. Swaters ${ }^{2}$, V. C. Rubin ${ }^{3}$, S. D'Odorico ${ }^{4}$, and S. S. McGaugh ${ }^{2}$ \\ ${ }^{1}$ Max-Plank-Institut für Extraterrestrische Physik, Giessenbachstraße, 85741 Garching bei München, Germany \\ e-mail: lcoccato@mpe.mpg.de \\ 2 Department of Astronomy, University of Maryland, College Park, MD 20742-2421, USA \\ 3 Carnegie Institution of Washington, 5241 Broad Branch Road NW, Washington, DC 20015, USA \\ ${ }_{4}^{4}$ European Southern Observatory, Karl-Schwarzschild-Straße 2, 85748 Garching bei München, Germany
}

Received 17 June 2008 / Accepted 12 September 2008

\begin{abstract}
Aims. We have studied the bulge and the disk kinematics of the giant low surface brightness galaxy ESO 323-G064 in order to investigate its dynamical properties and the radial mass profile of the dark matter (DM) halo.

Methods. We observed the galaxy with integral field spectroscopy (VLT/VIMOS, in IFU configuration), measured the positions of the ionized gas by fitting Gaussian functions to the [O III] $\lambda \lambda 4959,5007$ and $\mathrm{H} \beta$ emission lines, and fit stellar templates to the galaxy spectra to determine velocity and velocity dispersions. We modeled the stellar kinematics in the bulge with spherical isotropic Jeans models and explored the implications of self consistent and dark matter scenarios for NFW and pseudo isothermal halos.

Results. In the bulge-dominated region, $r<5^{\prime \prime}$, the emission lines show multi-peaked profiles. The disk dominated region of the galaxy, $13^{\prime \prime}<r<30^{\prime \prime}$, exhibits regular rotation, with a flat rotation curve that reaches $248 \pm 6 \mathrm{~km} \mathrm{~s}^{-1}$. From this we estimate the total barionic mass to be $M_{\mathrm{bar}} \sim 1.9 \times 10^{11} M_{\odot}$ and the total DM halo mass to be $M_{\mathrm{DM}} \sim 4.8 \times 10^{12} M_{\odot}$. The stellar velocity and velocity dispersion have been measured only in the innermost $\approx 5^{\prime \prime}$ of the bulge, and reveal a regular rotation with an observed amplitude of $140 \mathrm{~km} \mathrm{~s}^{-1}$ and a central dispersion of $\sigma=180 \mathrm{~km} \mathrm{~s}^{-1}$. Our simple Jeans modeling shows that dark matter is needed in the central $5^{\prime \prime}$ to explain the kinematics of the bulge, for which we estimate a mass of $\approx(7 \pm 3) \times 10^{10} M_{\odot}$. However, we are not able to disentangle different DM scenarios. The computed central mass density of the bulge of ESO 323-G064 resembles the central mass density of some high surface brightness galaxies, rather than that of low surface brightness galaxies.
\end{abstract}

Key words. galaxies: kinematics and dynamics - galaxies: spirals - galaxies: individual: ESO 323-G064

\section{Introduction}

Galaxy properties define a continuum, in size, luminosity, mass, and surface brightness. Low surface brightness (LSB) galaxies have significantly extended the range in surface brightness over which galaxies can be studied, and thus they have received a great deal of attention in studies of dark matter (e.g. Pfenniger et al. 1994; de Blok \& McGaugh 1997; McGaugh et al. 2001; Swaters et al. 2000, 2003; Kuzio de Naray et al. 2008), stellar content (e.g. de Blok et al. 1995; Bothun et al. 1997; Boissier et al. 2008) and gas content (e.g. O'Neil \& Schinnerer 2003; Pizzella et al. 2008b). Because of their unique properties, LSB galaxies also play a significant role in our understanding of the universe. Their distribution in the field and in galaxy clusters will help us to understand the bright and dark matter distribution in the local neighborhood and in the universe; their number and density distribution may be relevant to the study of damped Ly- $\alpha$ absorption seen against quasars (e.g. Jimenez et al. 1999; O'Neil 2002). Like high surface brightness galaxies, LSB galaxies also span a range in properties, such as size, mass, and bulge size. For example, Bothun et al. (1990) discovered a class of giant LSB galaxies, and Beijersbergen et al. (1999) studied a sample of bulge-dominated LSB galaxies. To date, very little is known

* Based on observations carried out at the European Southern Observatory (ESO 075.B-0695). of the properties of giant-sized or bulge-dominated LSB galaxies even though they can enhance our understanding of galaxy formation and evolution by providing an opportunity to sample galaxies in a hitherto almost unexplored regime of galaxy properties. Several scenarios have been proposed to explain their formation: density peak in voids (Hoffman et al. 1992), bar instability (Noguchi 2001; Mayer \& Wadsley 2004), or secular evolution from ring galaxies (Mapelli et al. 2008).

To fill the gap between regular and giant LSBs, Swaters, Rubin, \& McGaugh (SRM in preparation) have recently completed a study of the kinematics of a sample of bulge dominated LSB galaxies. Their aim is to study the dark matter properties and to determine whether bulge-dominated LSB galaxies are dark matter dominated like other LSB galaxies (de Blok \& McGaugh 1997; Swaters et al. 2003).

In this paper we show additional results obtained for the giant LSB galaxy ESO 323-G064, selected from the SRM project. ESO 323-G064 has an heliocentric velocity of $14830 \mathrm{~km} \mathrm{~s}^{-1}$ at a distance of $194 \mathrm{Mpc}$ (assuming $H_{0}=75 \mathrm{~km} \mathrm{~s}^{-1} \mathrm{Mpc}^{-1}$ and a galactocentric correction of $-278 \mathrm{~km} \mathrm{~s}^{-1}$ ). On its digital sky survey and 2MASS images, ESO 323-G064 has a bright, compact bulge, surrounded by a low surface brightness disk. There is some evidence of a weak bar component. The long slit spectra of ESO 323-G064 obtained (SRM, in preparation) with the $6.5 \mathrm{~m}$ Baade Telescope at LCO, show strong, double-peaked emission 
from $\mathrm{H} \alpha,[\mathrm{N} \mathrm{II}],[\mathrm{SII}],[\mathrm{OI}]$ in the nuclear region. The large extent $(47 \mathrm{kpc})$ of the $\mathrm{H} \alpha$ and the large peak-to-peak rotation velocity of $445 \mathrm{~km} \mathrm{~s}^{-1}$ (on the plane of the sky) mark ESO 323-G064 as a likely giant LSB.

In order to determine the kinematics of the stars in the bulgedominated region, and to compare these motions with those of the gas in both the nucleus and at larger radii, we decided to obtain two dimensional data using an integral field unit. This will give a better picture of the complex gaseous kinematics especially the double peaked emission revealed by the long slit data. Optical two-dimensional observations in LSB galaxies have been obtained in the past (i.e. Swaters et al. 2003; Kuzio de Naray et al. 2006, 2008; Pizzella et al. 2008b) but, so far, never for the stellar component of a giant LSB.

The paper is organized as follow: in Sect. 2 we present the integral field observations and discuss the data reduction process; in Sects. 3 and 4 we present the kinematical results for the ionized gas and stellar components respectevely; in Sect. 5 we present the Jeans model to the stellar kinematics and in Sect. 6 we discuss the results.

\section{Observations and data reduction}

The integral-field spectroscopic observations were carried out in service mode with the Very Large Telescope (VLT) at the European Southern Observatory (ESO) in Paranal (Chile) from June to August 2005 during dark time. The Unit Telescope 3 (Melipal) was equipped with the Visible Multi Object Spectrograph (VIMOS) in the Integral Field Unit (IFU) configuration. The seeing, measured by the ESO Differential Image Meteo Monitor, was generally below $0 . ' 7$ except for observations on July 1st during which the seeing was around 1.'5 (one 30 min exposure) and observations on 4th August in which the seeing was between 0.'7 and 1.'0 (one 30 min exposure).

The observations were organized into 8 exposures of $30 \mathrm{~min}$ each, divided into two different pointings with an offset of 25 arcsec. Each telescope pointing has a field of view of $27 \times$ $27 \operatorname{arcsec}^{2}$ and it is recordered on a 4 CCD mosaic. We hereafter refer to the 4 CCDs as quadrants, and we refer to the 2 observed fields as field A and field B. Quadrants \# 1,2,3 and 4 cover the NE,SE,SW and NW portions of the field of view respectively. Field A $(4 \times 30$ min exposures, with a few pixel dithering) covers the NW side of the galaxy, while Field B $(4 \times 30$ min exposures with a few pixel dithering) covers the SE side of the galaxy. The field of view of the four VIMOS quadrants was projected onto a micro lens array. This was coupled to optical fibers which were rearranged on a linear set of micro lenses to produce an entrance pseudo-slit to the spectrograph. The pseudo-slit was 0 .' $^{\prime} 95$ wide and generated a total of 1600 spectra covering the field of view with a spatial resolution of 0.'67 per fiber. Each quadrant was equipped with the HR-blue resolution grism (4120-6210 А) and a thinned, back-illuminated EEV44 CCD with $2048 \times 4096$ pixels of $15 \times 15 \mu \mathrm{m}^{2}$. The spectral resolution measured on the sky emission lines is $\lambda / \Delta \lambda \approx 1700 ; \sigma_{\text {instr }} \approx 75 \mathrm{~km} \mathrm{~s}^{-1}$.

Together with every exposure a set of night calibration spectra were taken: one comparison spectrum (Neon plus Argon) for the wavelength calibration and 3 quartz lamp exposures for the flat field correction and fibers identification.

For each VIMOS quadrant all the spectra were traced, identified, bias subtracted, flat field corrected, corrected for relative fiber transmission, and wavelength calibrated using the routines of the ESO Recipe EXecution pipeline (ESOrex) ${ }^{1}$. Cosmic rays and bad pixels were identified and cleaned using standard MIDAS ${ }^{1}$ routines. We checked that the wavelength rebinning was done properly by measuring the difference between the measured and predicted wavelength for the brightest nightsky emission lines in the observed spectral ranges (Osterbrock et al. 1996). The resulting accuracy in the wavelength calibration is better than $5 \mathrm{~km} \mathrm{~s}^{-1}$. The intensity of the night-sky emission lines was used to correct for the different relative transmission of the VIMOS quadrants. The processed spectra were organized in a data cube using the tabulated correspondence between each fiber and its position in the field of view. From the single exposures of every observed field we built a single data cube. The spectra were co-added after correcting for the position offset. The offset was determined by comparing the position of the intensity peaks of the two reconstructed images obtained by collapsing the data cubes along the wavelength direction. The accuracy of the offset is \pm 0.5 pixel $(\simeq 0$ ' 33 ). This slight deterioration of the spatial resolution does not affect the results.

Finally, we co-added the two available data cubes (field A and field B) by using the intensity peaks of the flux maps as a reference for the alignment. In this way we produced a single data cube to be analyzed in order to derive the surface brightness and two dimensional field kinematics.

In Fig. 1 we show the two reconstructed images of both the observed fields, obtained by collapsing the data cube along the direction of dispersion.

\subsection{Sky subtraction}

The sky subtraction is a critical step in the data analysis (especially for stellar kinematics) because 1) the galaxy absorption lines are weak and 2) some weak sky emission lines overlap with the galaxy emission lines.

The automatic ESOrex pipeline evaluated and corrected the spectra for the different efficiency of the fibers and the 4 detectors. But in order to avoid possible problems due to a poor correction of the efficiency difference for the four quadrants, we decided to evaluate the sky contribution for every quadrant separately. This choice is supported by the fact that the useful signal for the stellar kinematics is contained entirely in one quadrant (quadrant \#3 for field A observations and quadrant \#4 for field B observations, respectively).

For each quadrant we identified the spectra in which the galaxy's contribution is negligible and we computed the median. Finally we subtracted the median sky spectrum from every single spectrum of the quadrant.

\section{Gaseous kinematics}

ESO 323-G064 presents very bright emission lines in the nucleus together with fainter emission lines located at larger radii.

The gas kinematics are measured by fitting to each spectrum a background level and several Gaussian components. The number of components is chosen depending on which and how many emission lines are visible at the considered spectrum. No constraints are applied to the fitting parameters: velocities, velocity dispersions and intensities are independent.

To determine the kinematics, we used a non-linear leastsquares minimization algorithm based on the robust LevenbergMarquardt method implemented by Moré et al. (1980). The

\footnotetext{
1 ESOrex and MIDAS are developed and maintained by the European Southern Observatory.
} 

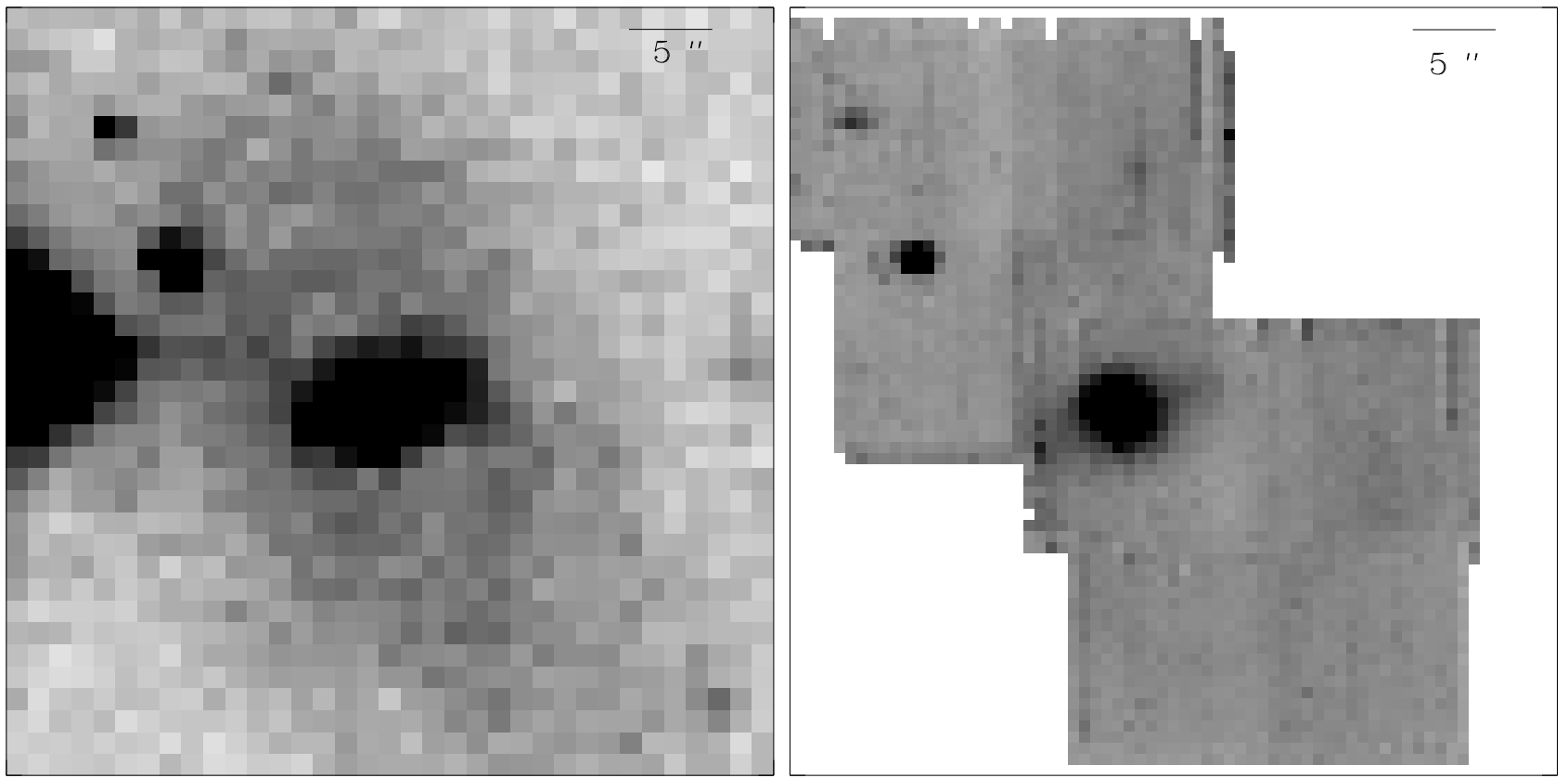

Fig. 1. Left panel: $R$-band image of ESO 323-G064 from the ESO-Uppsala Galaxy surface photometry catalog (ESO-LV, Lauberts 1982). North is top, east is left. Right panel: reconstructed image of ESO 323-G064 from combination of 8 exposures. Scale and orientation are as in the left panel.
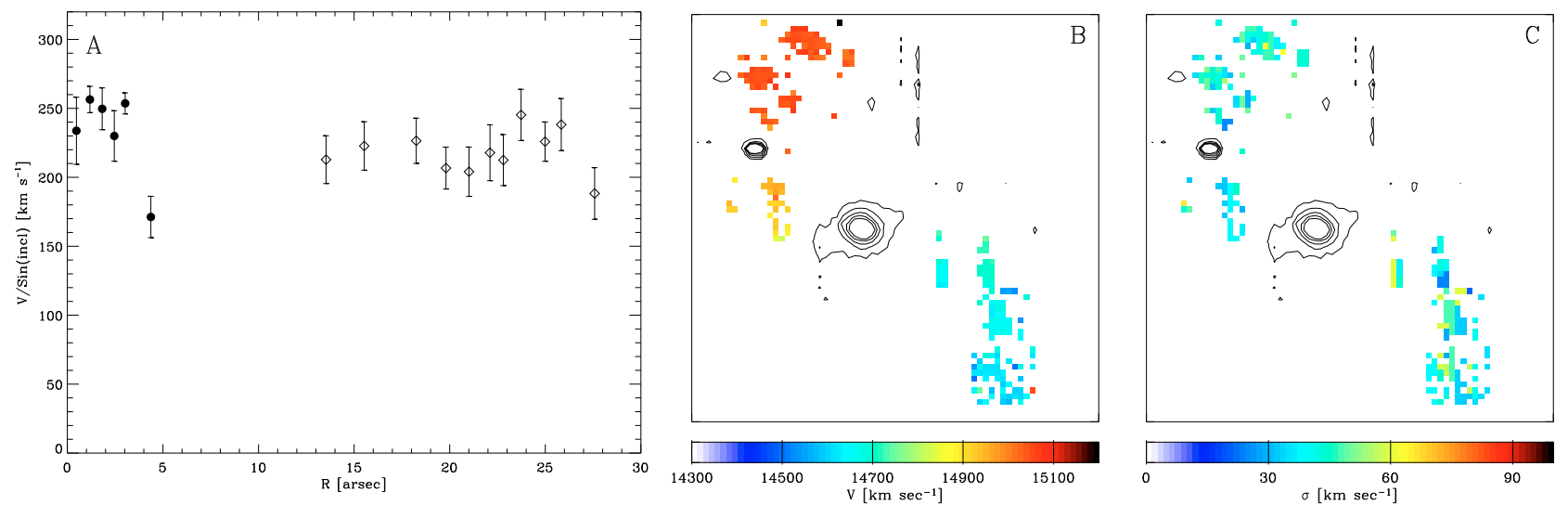

Fig. 2. A) Rotation curve of ESO 323-G064 from $\mathrm{H} \beta$ emission, formed from the two dimensional velocity field of the disk (open diamonds). Filled circles are the stellar rotation circular velocity curve after correction for asymmetric drift (see Sect. 5.4.1). B) Velocity field of $\mathrm{H} \beta$ emission at large nuclear distance. C) Velocity dispersion field of $\mathrm{H} \beta$ emission at large nuclear distance. In both panels B) and C), galaxy central contours are shown, the field of view is $46^{\prime \prime} \times 46^{\prime \prime}$, North is at top, East at left.

actual computation has been done using the MPFIT procedure implemented by C. B. Markwardt under the IDL environment ${ }^{2}$.

\subsection{Ionized gas at large radii}

Faint $\mathrm{H} \beta$ emission is detected at $15^{\prime \prime} \leq r \leq 35^{\prime \prime}$. At this distance from the center, the gas is associated with the disk component. It reaches $\simeq 15050 \mathrm{~km} \mathrm{~s}^{-1}$ on the NE side and $\simeq 14500 \mathrm{~km} \mathrm{~s}^{-1}$ on the SW side. The intrinsic velocity dispersion ranges between 30 and $50 \mathrm{~km} \mathrm{~s}^{-1}$ showing that this gaseous component is quite cold (see Fig. 2).

To derive a rotation curve from the two-dimensional velocity field of the gaseous disk, we divided the gaseous disk in concentric rings, with the same inclination $(i)$ and the same orientation

\footnotetext{
2 The updated version of this code is available on http://cow. physics.wisc. edu/ craigm/idl/idl.html
}

on the sky (PA). Each ring is constructed in order to contain the same numbers (20) of data points, with velocities $v_{n}$ and position angles $\phi_{n}{ }^{3}$. We also assume the gas is moving in circular motion, therefore the velocity $V_{M}(R)$ in the $M$ th bin centered at $R$ is measured by fitting the following function to the $N=20$ points within the bin:

$V(\phi, R)=V_{M}(R) \times \cos (\phi+\mathrm{PA}) \times \sin (i)+V_{\mathrm{sys}}$.

The best fit results in a systemic velocity of $V_{\text {sys }}=14830 \pm$ $12 \mathrm{~km} \mathrm{~s}^{-1}$, a position angle on the sky of PA $=38 \pm 3$ (slightly different from the 32 degrees derived on the ESO-LV $R$-band and $B$-band images, an inclination of $i=61.5 \pm 0.9$ (consistent with

\footnotetext{
${ }^{3} \phi_{n}$ are measured counterclockwise starting from the galaxy major axis.
} 
the 60 degrees from ESO-LV images ${ }^{4}$.) and a center consistent within a pixel with the photometric center of the bulge. At every bin position the IDL fitting procedure gives the amplitude of rotation $V_{M}(R)$ and its error. We show in the left panel of Fig. 2 the derived rotation curve of ESO 323-G064. The rotation curve is relatively constant with radius. The average rotation value (taking into account the incliation) is $248 \pm 6 \mathrm{~km} \mathrm{~s}^{-1}$. The result does not change if we hold the position angle constant at PA $=32$ as derived from ESO-LV images.

Since the velocity dispersion of the ionized gas is quite small, we can approximate the measured velocity rotation with the circular velocity $V_{\mathrm{C}}$. This allows us to estimate the total baryonic mass of the galaxy, from the baryonic Tully Fisher relation, as described in McGaugh (2005):

$M_{\mathrm{bar}}=50 \cdot V_{\mathrm{C}}^{4}=(1.9 \pm 0.2) \times 10^{11} M_{\odot}$.

In addition, we can also make a rough estimate of the total mass $M_{\mathrm{DM}}$ of the dark matter halo through the relation:

$M_{\mathrm{DM}}=2.52 \times 10^{12}\left(\frac{V_{\mathrm{C}}}{200}\right)^{3}=(4.8 \pm 0.4) \times 10^{12} M_{\odot}$.

To derive the formula, we followed the prescriptions by Bryan $\&$ Norman (1998) and Bullock et al. (2001) using $\Omega_{\Lambda}=0.7$, $\Omega_{m}=0.3, h=0.7, \Delta_{c}=105.4$ and $z \approx 0.05$. The error estimates for $M_{\mathrm{bar}}$ and $M_{\mathrm{DM}}$ are simply given by the error propagation in Eqs. (2) and (3).

\subsection{Ionized gas in nuclear region}

$\mathrm{H} \beta$, [O III] $\lambda 4959$ and [O III] $\lambda 5007$ bright emission lines are visible in the nuclear regions within $r<5^{\prime \prime}$. Interestingly, at some locations these emission lines are triple peaked (see Figs. 3 and 4). This complexity is probably generated by three barely resolved emission-line regions located close to the center but at different azimuthal angles.

In order to have kinematical informations for all the 3 regions, we fitted each of the the galaxy emission lines with three independent Gaussian functions, and the results (velocity, velocity dispersion and intensity) were compiled separately.

The velocity field of the three components do not show a regular rotation. Their averaged radial velocities are approximately $14539 \pm 38,14726 \pm 31$, and $14992 \pm 26 \mathrm{~km} \mathrm{~s}^{-1}$. These values were inferred from the [O III] velocity field (mean value between [O III] $\lambda 4959$ and [O III] $\lambda 5007$ ) because it is not affected by stellar absorption as is the $\mathrm{H} \beta$ emission line. The velocity dispersion in each of the 3 regions is between 50 and $100 \mathrm{~km} \mathrm{~s}^{-1}$.

The measured FWHM of the 3 regions is about 1."7, which is very similar to the one of the foreground star $\left(\approx 11^{\prime \prime} 3\right)$. The maximum distance measured between the centroids of the 3 regions is 1 .'0.

We can exclude that the observed multi-peak line profile is an artifact caused by the data reduction because:

1. The sky lines observed in each quadrant (either in the raw spectra or in the final reduced data cube) are not multi peaked. This eliminates instrumental effects (e.g., a shift of the instrument during the exposure) or pipeline errors (e.g., a shift in the dispersion direction during the data cube combining) as a source of the triple-peaked profiles.

\footnotetext{
${ }^{4}$ Images from scanned plates can be downloaded from

http://www . astro-wise.org/portal/aw_datasources.shtml.
}

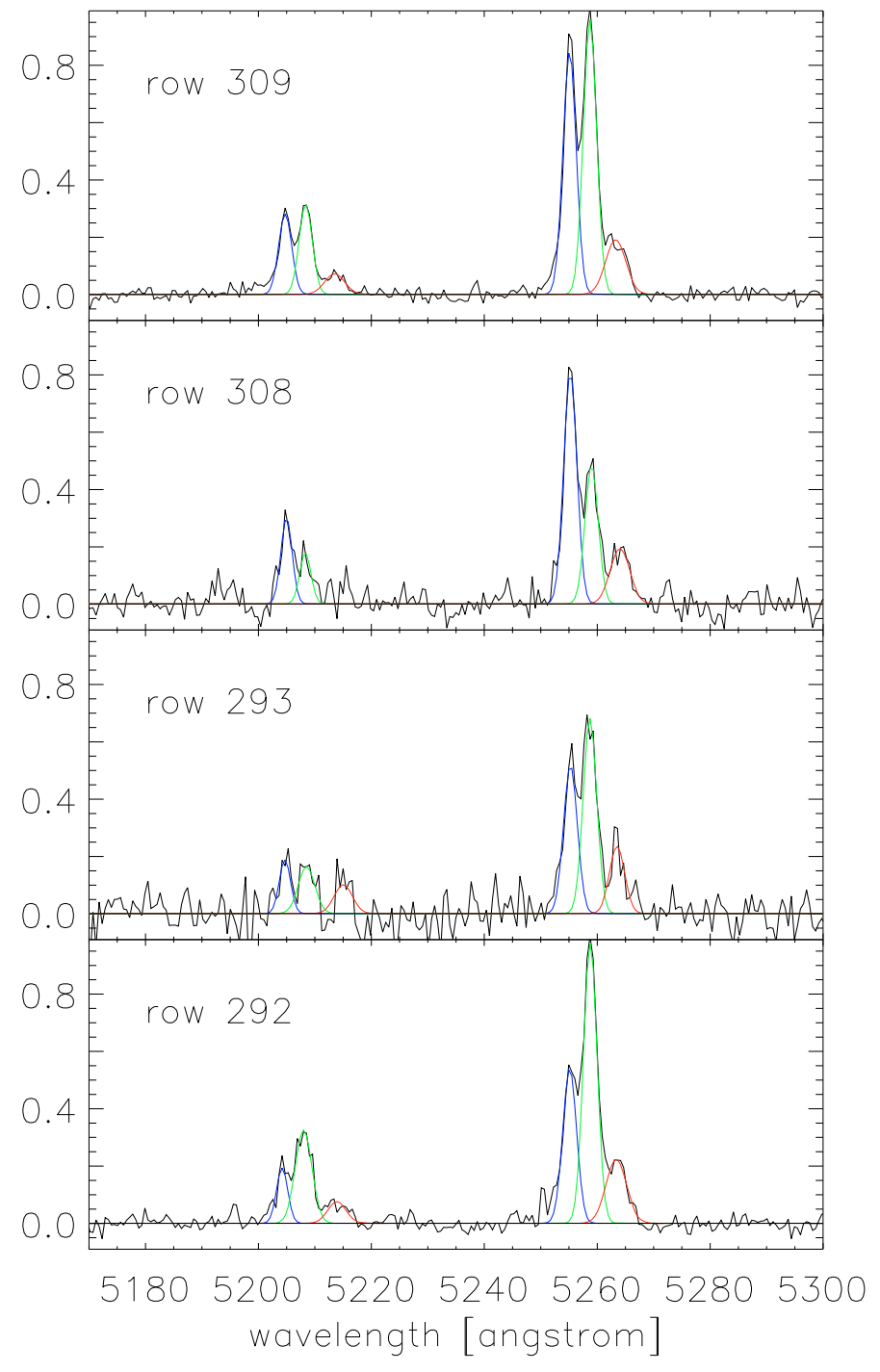

Fig. 3. Examples of [O III] emission line profiles, in which the 3 peaks are visible. $\mathrm{H} \beta$ emission line has been omitted in this plot because it is very faint with respect to the [O III]. The blue, green and red lines represent the fits to the first, second and third emitting regions respectively.

2. The three peaks in the galaxy emission line are present before and after the sky subtraction and they are present also in the raw spectra. Moreover, the few sky lines which are overlap with the galaxy emission lines are very weak.

3. The three peaks are visible in all the galaxy emission lines and the ratio between the intensities of the components is (almost) the same if measured in $\mathrm{H} \beta$, [O III] $\lambda 4959$ or [O III] $\lambda 5007$.

4. The peaks are visible also if we consider only the exposure with the best seeing condition.

\section{Stellar kinematics}

The stellar kinematics have been measured by means of the Penalized Pixel-Fitting (ppxf) method by Cappellari \& Emsellem (2004). We chose a library of stellar templates from Valdes et al. (2004) provided together with the ppfx tools. These spectra have a spectral range of 4780-5460 $\AA$ and spectral resolution of $1.0 \AA$ at $5100 \AA\left(\sigma \sim 22 \mathrm{~km} \mathrm{~s}^{-1}\right.$, which had been deteriorated in order to match the instrumental one). 

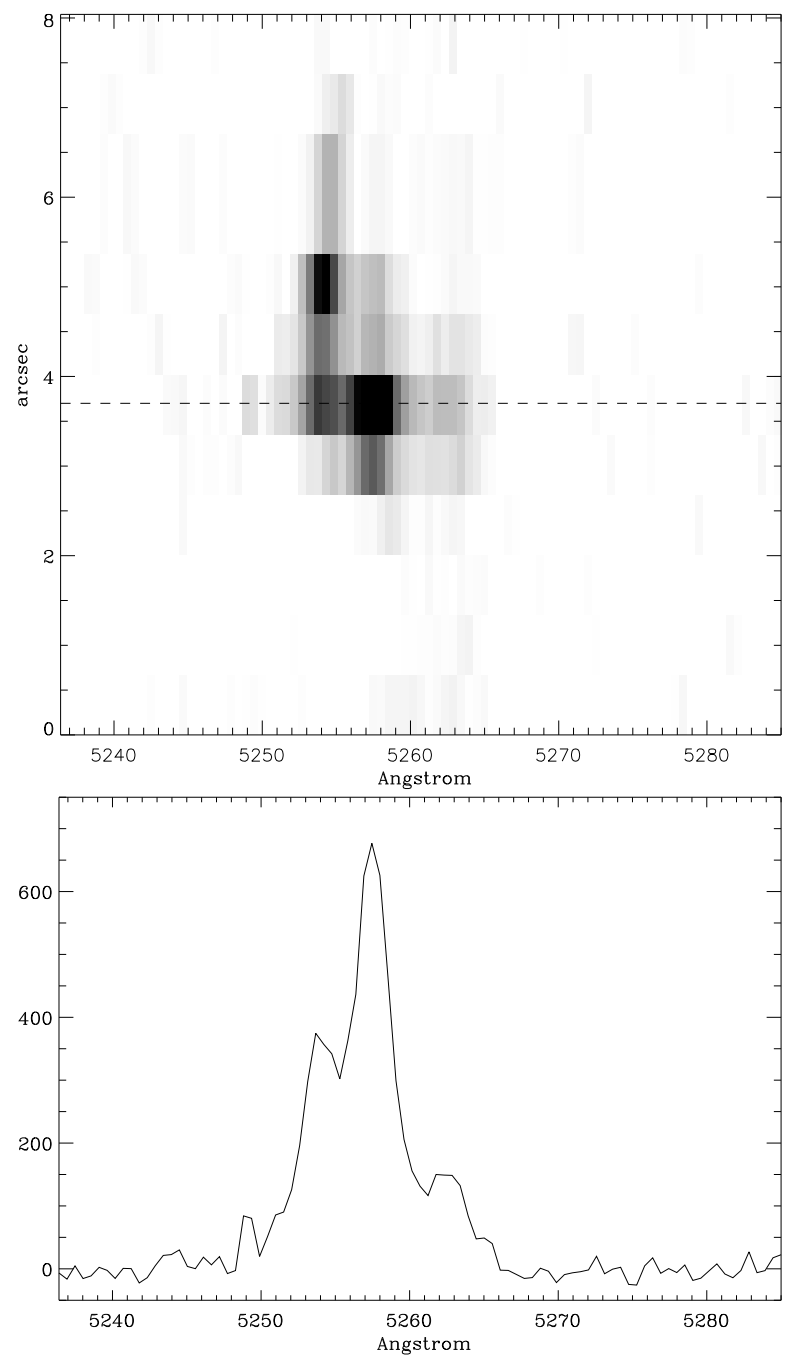

Fig. 4. Upper panel: position velocity diagram towards the galactic nucleus extracted along the major axis. Lower panel: intensity profile of the PV diagram along the dashed line in the upper panel. The 3 peaks of the emission line are clearly visible.

The stellar templates from this library were choosen among type $\mathrm{K}$ stars, which are commonly used as kinematical templates.

Errors of the measurements ranged around $15-20 \mathrm{~km} \mathrm{~s}^{-1}$. We did several tests using additional templates from the same library and from the MILES library (Sánchez-Blázquez et al. 2006). Results differ from the original values with a scatter of $10 \mathrm{~km} \mathrm{~s}^{-1}$, which is consistent with the measurement errors.

No multiple peaked features in the absorption line spectra are visible at our spectral resolution (and at our signal to noise ratio). Some examples of the fit results are given in Fig. 5 and the two-dimensional velocity and velocity dispersion fields are shown in Fig. 6 . The velocity of the stars ranges from $\sim 14700$ to $\sim 14900 \mathrm{~km} \mathrm{~s}^{-1}$, and a clear pattern of rotation is visible.

The central intrinsic velocity dispersion within $1^{\prime \prime}$ is $\approx 180 \pm$ $10 \mathrm{~km} \mathrm{~s}^{-1}$, which, combined with the gaseous circular velocity value $V_{\mathrm{C}}=248 \mathrm{~km} \mathrm{~s}^{-1}$ (derived in Sect. 3.1), set this galaxy close the $\left(V_{\mathrm{C}}-\sigma_{\mathrm{c}}\right)$ relation for LSB galaxies (Courteau et al. $2007 \mathrm{a}, \mathrm{b})$. According to these authors, the predicted $V_{\mathrm{C}}$ value for a bulge with $\sigma_{\mathrm{c}}=180 \mathrm{~km} \mathrm{~s}^{-1}$ is $V_{\mathrm{C}} \approx 290 \mathrm{~km} \mathrm{~s}^{-1}$.
The $V / \sigma$ of the bulge is computed using the formalism introduced by Binney (2005) and applied by Cappellari et al. (2007).

$$
\begin{aligned}
\left(\frac{V}{\sigma}\right)_{\mathrm{INTR}} & =\left(\frac{V}{\sigma}\right)_{\mathrm{OBS}} / \sin (i) \\
& =\frac{1}{\sin (i)} \sqrt{\frac{\sum_{n=1}^{N} F_{n} V_{n}^{2}}{\sum_{n=1}^{N} F_{n} \sigma_{n}^{2}}} \approx 0.56 \pm 0.02
\end{aligned}
$$

where $F_{n}, V_{n}$ and $\sigma_{n}$ are the flux (extrapolated from the surface brightness profile), velocity and velocity dispersion measured from the $n$th spectra. An inclination of 62 degrees and isotropy assumption are adopted to evaluate the measurements in the edge-on case. Errors are calculated using classic formulas for error propagation.

This value is consistent with the predicted value of an edgeon isotropic oblate system (Binney 1978) $0.4<\left(\frac{V}{\sigma}\right)_{\text {INTR }}<0.6$ for an intrinsic ellipticity ranging $0.13<\epsilon_{\text {INTR }}<0.27$. The intrinsic value for ellipticity has been calculated from the observed one $\left(0.1<\epsilon_{\mathrm{OBS}}<0.2\right.$, Sect. 5.1) after correction for inclination using the formula (see Binney \& Tremaine 1987):

$\epsilon_{\mathrm{INTR}}=1-\sqrt{1+\epsilon_{\mathrm{OBS}}\left(\epsilon_{\mathrm{OBS}}-2\right) / \sin (i)}$.

\section{Stellar dynamical modeling}

In this section we present a simple dynamical model of the stellar velocity and velocity dispersion fields ${ }^{5}$.

We assume the total luminous plus dark matter mass distribution of the central galaxy bulge is spherical. The low reported value for the ellipticity (Sect. 5.1) allows us to make this assumption. In addition we assume isotropy for the bulge, given the fact that the inferred $V / \sigma$ is consistent with an isotropic rotator, as shown in Sect. 4.

The main deviation from spherical symmetry in the bulge of ESO 323-G064 is the bar component, clearly visible in Fig. 1. Given the limited amount of stellar kinematic data in the bulge, it is not possible to take into account the complexity of stellar motions in the bar potential. However, from the isophotes shown in Fig. 6 it is evident that the bar contribution dominates only the outer regions of the velocity field, for $R \geq 3$ ". 5 . Stellar orbits in those regions will be elongated towards the bar direction, which is nearly orthogonal to the kinematic major axis. This will lead to an underestimation of the stellar rotation velocity and it will be quantified in Sect. 5.4.

We assume also that the disk contribution to the total mass in the bulge region (inner $5^{\prime \prime}$ ) is negligible. We do not have direct measurement of the stellar disk kinematics, but its low luminosity (compared to the bulge) supports our assumption. Moreover, several studies demonstrated that the disk contribution to the inner dynamics in low surface brightness galaxies is almost insignificant (Swaters et al. 2000, 2003).

The limited spatial extent of the observations and the lack of good photometry for this galaxy do not allow us to go in more detail.

The Jeans equation in radial coordinates for such a system is (we followed the formalism adopted by Hui et al. 1995 and Peng et al. 2004):

$\frac{\rho_{k}(r) \sigma_{\mathrm{int}}^{2}(r)}{r}=-\frac{G M(r) \rho_{k}(r)}{r^{2}}+\frac{\rho_{k}(r) V_{\mathrm{rot}}^{2}(r)}{r}$

5 We assume $H_{0}=75 \mathrm{~km} \mathrm{~s}^{-1} \mathrm{Mpc}^{-1}$ in the modeling. 

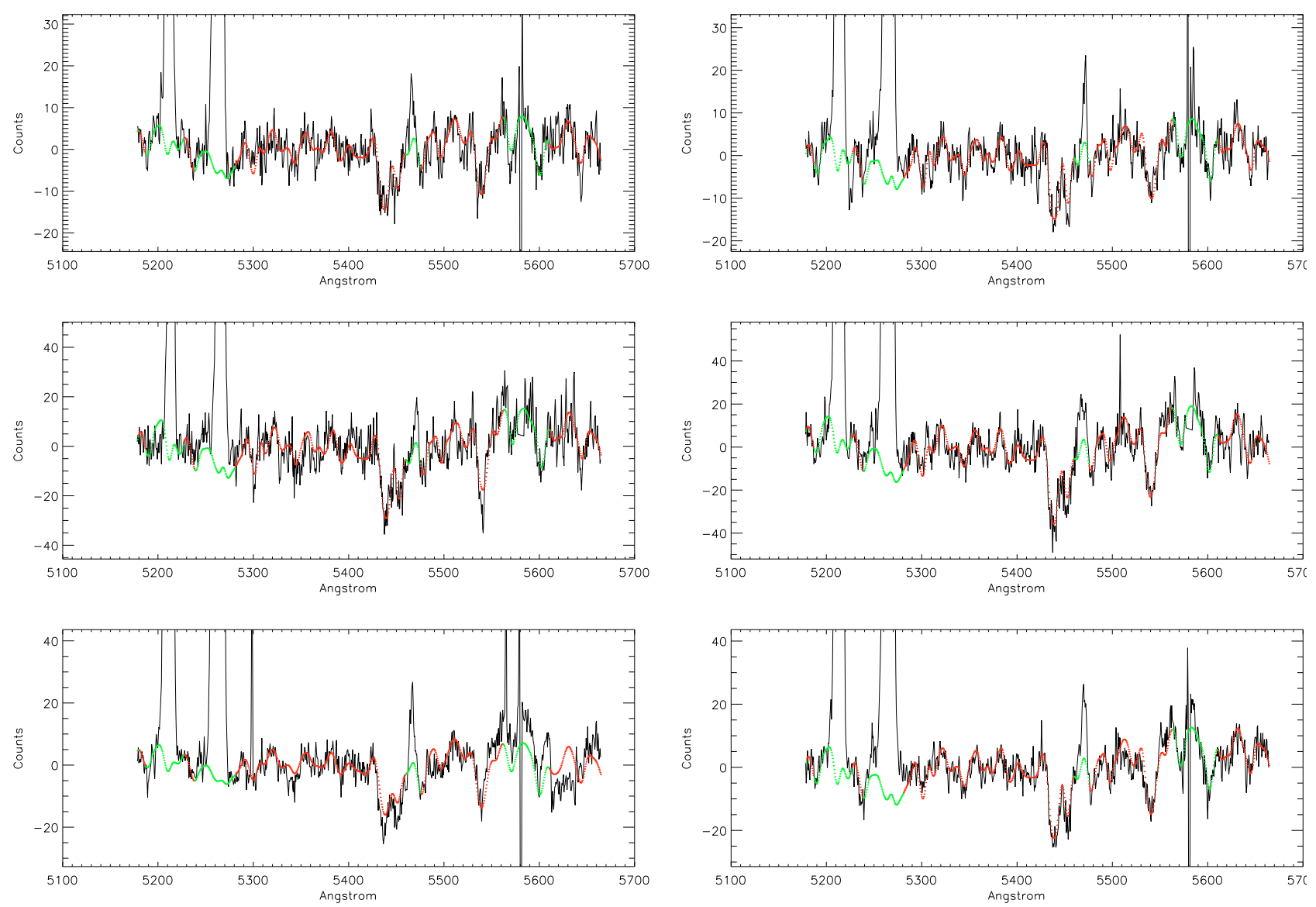

Fig. 5. Examples of the stellar kinematics fit quality. Black is the galaxy spectrum, while red (green) represents the portion of the stellar template which was included (excluded) from the fit.

which, when solved for the intrinsic velocity dispersion $\sigma_{\text {int }}$, is:

$\sigma_{\text {int }}^{2}(r)=\frac{1}{\rho_{k}(r)} \int_{r}^{\infty} \rho_{k}(x) \frac{G M(x)-x V_{\text {rot }}^{2}(x)}{x^{2}} \mathrm{~d} x$

where $\rho_{k}$ is the mass density radial profile for the tracer of the potential (i.e. the stars), $V_{\text {rot }}$ is the intrinsic rotation curve of the stellar component, $M$ is the total mass of the galaxy and $G$ is the gravitational constant.

The velocity dispersion projected on the sky is:

$\sigma^{2}(R)=\overline{V_{\mathrm{LOS}}^{2}}(R)-V_{\mathrm{s}}(R)^{2}$

where $V_{\mathrm{s}}$ is the projected rotation curve of the stars (see Sect. 5.2) and $\overline{V_{\text {LOS }}}$ is the line-of-sight second velocity moment, projected into the sky, given by:

$\overline{V_{\mathrm{LOS}}^{2}}(R)=\frac{2 R}{\Sigma(R)} \int_{R}^{\infty}\left(\sigma_{\mathrm{int}}^{2}(r)+V_{\mathrm{rot}}^{2}(r) \frac{R^{2}}{r^{2}}\right) \frac{\rho_{k}(r) r}{\sqrt{r^{2}-R^{2}}} \mathrm{~d} r$.

\subsection{Density of the kinematical tracer}

There are no good CCD images of ESO 323-G064 available in the literature. The better available photometry is from the ESOLV catalog 6 . We performed a bulge/disk decomposition using galfit (Peng et al. 2002), adopting a de Vaucouleurs law for the bulge and an exponential law for the disk. We derived an

${ }^{6}$ See Sect. 3.1 for the images source. effective radius $R_{\mathrm{e}}=0 .{ }^{\prime} 67$ and an average ellipticity of 0.3 for the bulge, position angle PA $=32$ and inclination $i=60$ for the disk. With the ESO-LV images we confirmed also that the stellar disk of ESO 323-G064 is in the LSB regime. Its surface brightness in the B-band ranges from $\sim 23.3 \mathrm{mag} \operatorname{arcsec}^{-2}$ at the center to $\sim 26.5 \mathrm{mag} \operatorname{arcsec}^{-2}$ at $35^{\prime \prime}$. Even though the ESO-LV images have been useful to get an estimate of the surface brightness, they had insufficient signal-to-noise and spatial resolution to get reliable values of the bulge parameters. We therefore used our VIMOS observations, collapsing the observed data cube along the dispersion direction and deriving a surface brightness profile from the resulting image. We fit a de Vaucouleurs law to this profile and derived an effective radius $R_{\mathrm{e}}=0.9$ (see Fig. 7) and a ellipticity ranging from 0.1 to 0.2 , not too far from the adopted spherical approximation. Although the signal-to-noise ratio of the VIMOS observations is low, the advantage is that the VIMOS spatial resolution (0.'67/pixel) is higher than that of the ESO-LV images (1".35/pixel). This makes us more confident of bulge parameter values based on VIMOS observations than values based on ESO-LV images.

The de Vaucouleurs profile resembles the Hernquist (1990) mass model in which the intrinsic density distribution is given by:

$\rho_{k}(r)=\frac{M_{\mathrm{L}} a}{2 \pi} \frac{1}{r(r+a)^{3}}$

where $M_{\mathrm{L}}$ is the total luminous mass and $a=R_{\mathrm{e}} / 1.8153$. 

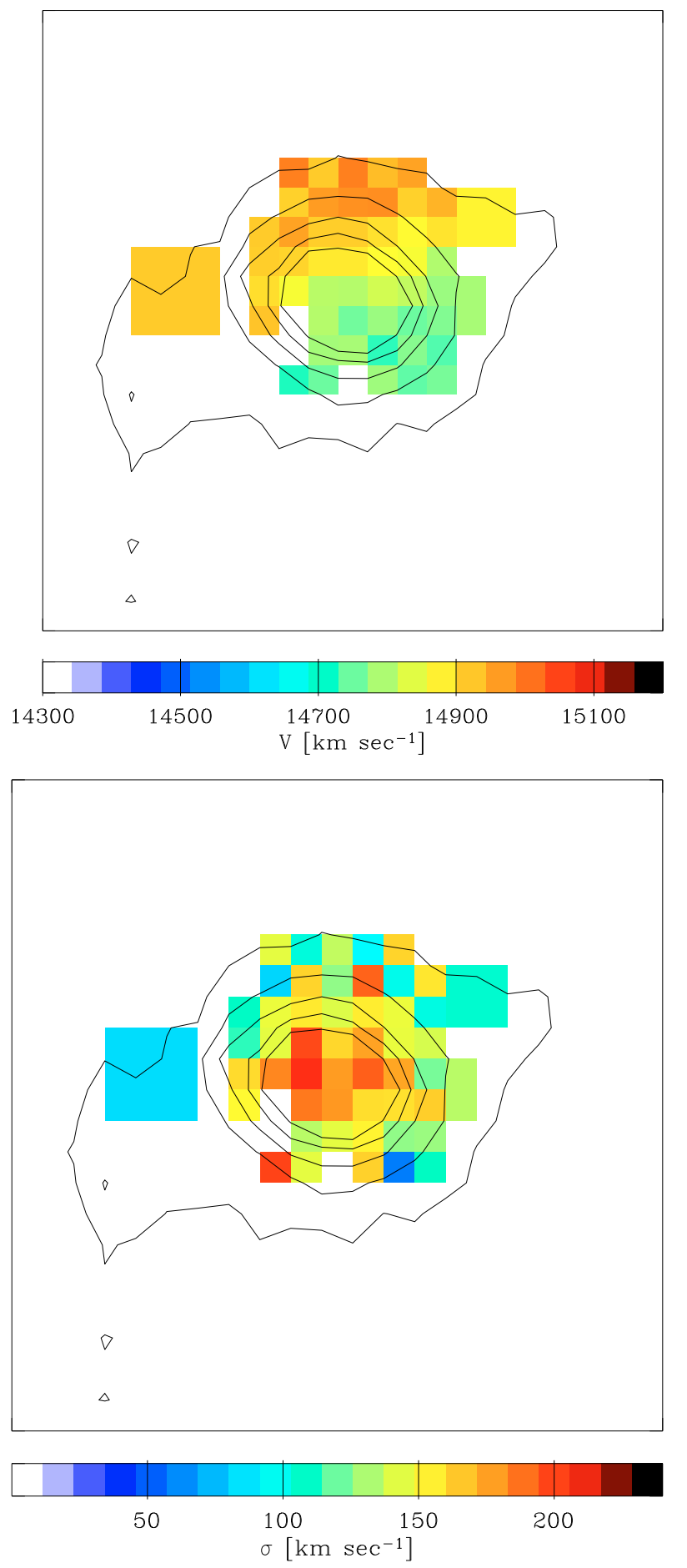

Fig. 6. Stellar radial velocity and velocity dispersion fields, with the bulge central isophotes shown. The field of view is $14^{\prime \prime} \times 14^{\prime \prime}$, North is up, East is left.

The projection on the sky of Eq. (10) is given by:

$\Sigma(R)=2 \int_{R}^{\infty} \frac{\rho_{k}(r) r}{\sqrt{r^{2}-R^{2}}} \mathrm{~d} r$.

Equations (10) and (11) will be used in Eq. (9) to determine the velocity moments for the mass calculation.

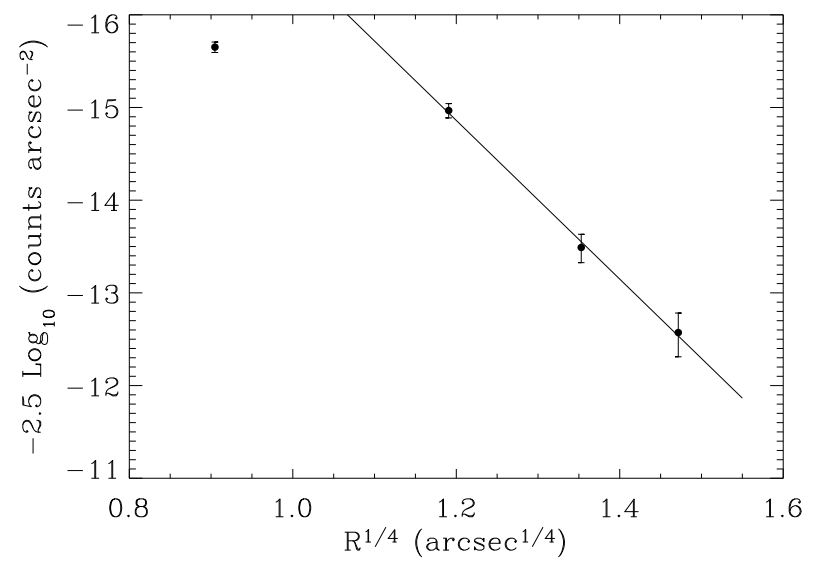

Fig. 7. Dots: surface brightness profile obtained by collapsing the data cube along dispersion direction. Continuous line: de Vaucouleurs fit to the measured surface brightness, with $R_{\mathrm{e}}=00^{\prime} 9$ and $\mu_{\mathrm{e}}=$ $-16.8 \mathrm{mag} \operatorname{arcsec}^{-2}$ (arbitrary zero point). The innermost point was not included in the fit, since it is affected by the seeing.

\subsection{Intrinsic and projected rotation curve}

Because of the limited spatial extent and resolution of the observations, a "bidimensional" approach to the Jeans equation cannot be reliably carried out. We therefore decided to average azimuthally and then bin radially the bidimensional velocity and velocity dispersion fields. To do that, we divided them in $N$ radial bins ${ }^{7}$ (after several tests, it turned out that $1^{\prime \prime} .5$ per bin is an optimal value) and for each radial bin at a given position $r_{N}$ we:

- calculated the velocity $V\left(r_{N}\right)$ by fitting the function $v^{\prime}(\phi)=$ $V\left(r_{N}\right) \times \cos (\phi+\mathrm{PA})+V_{\text {sys }}$ to all the velocity data points within the $N$ th bin. $\phi$ is the angle measured in the galaxy equatorial plane, PA is the galaxy position angle on the sky ${ }^{8}$ and $V_{\text {sys }}=14825 \mathrm{~km} \mathrm{~s}^{-1}$ is the adopted systemic velocity;

- calculated the velocity dispersion $\sigma_{N}\left(r_{N}\right)$ by averaging all the velocity dispersion data points within the $N$ th bin. In this computation, we took into account also the value of the velocity gradient along the resolution element ( $\left.11^{\prime \prime} 5\right)$ and removed it from the velocity dispersion.

In Eq. (7), the quantity $V_{\text {rot }}$ is the intrinsic stellar rotation curve. It can be parametrized with the expression:

$$
V_{\mathrm{rot}}(r)=\frac{v_{\infty} r}{\sqrt{r^{2}+r_{\mathrm{h}}^{2}}}
$$

where $v_{\infty}$ is its asymptotic value for the velocity and $r_{\mathrm{h}}$ is a scale parameter. The adopted function was chosen in order to properly match the observed velocity curve with the minimum number of free parameters. This expression has been used for example also in the spherical PNe system of Centaurus A (Hui et al. 1995; Peng et al. 2004).

The observed rotation curve is obtained projecting Eq. (12) on to the sky (Binney \& Tremaine 1987):

$V_{\mathrm{s}}(R)=\frac{2 R}{\Sigma(R)} \int_{R}^{\infty} \frac{\rho_{k}(r) V_{\text {rot }}(r)}{\sqrt{r^{2}-R^{2}}} \mathrm{~d} r$.

\footnotetext{
7 Bins have an elliptical shape with $\langle\epsilon\rangle=0.15$ in order to take into account the bulge ellipticity we observed.

${ }^{8}$ We fixed the position angle to the value PA $=38$ derived in Sect. 3.1 . We tested this assumption leaving PA free to vary in the fit and we found the value to be consistent with $38^{\circ}$.
} 
Equations (12) and (13) will be used in Eqs. (8) and (9) to compute the velocity moments.

\subsection{Radial mass profile}

The radial distribution of the total mass $M(r)$ of the galaxy bulge is given by the sum of the luminous and dark matter contents ${ }^{9}$. The contribution $M_{k}$ of the luminous component is obtained by integrating Eq. (10) in the volume. It leads to:

$M_{k}(r)=\frac{M_{\mathrm{L}} r^{2}}{(r+a)^{2}}$.

Together with the self consistent case, in which the total mass of the galaxy is given only by the contribution of the stars, we explored also two different scenarios for the dark matter content: Navarro et al. (1997, NFW hereafter), and the pseudo isothermal halos.

Their mass density distribution are:

$\rho_{\mathrm{NFW}}(r)=\frac{\rho_{\mathrm{s}}}{r / r_{\mathrm{S}}\left(r / r_{\mathrm{s}}+1\right)^{2}}$

$\rho_{\text {isot }}(r)=\frac{\rho_{0}}{1+\left(r / r_{0}\right)^{2}}$

where $r_{\mathrm{s}}, \rho_{\mathrm{s}}, r_{0}$ and $\rho_{0}$ are scale parameters.

The corresponding mass distributions are given by the volume integration of Eqs. (15) and (16). We obtain:

$M_{\mathrm{NFW}}(r)=4 \pi \rho_{\mathrm{s}} r_{\mathrm{s}}^{3}\left[\ln \left(1+r / r_{\mathrm{s}}\right)-\frac{r / r_{\mathrm{s}}}{1+r / r_{\mathrm{s}}}\right]$

$M_{\text {isot }}(r)=4 \pi \rho_{0} r_{0}^{3}\left[r / r_{0}-\arctan \left(r / r_{0}\right)\right]$.

In the dark matter scenarios, the total mass $M(r)$ is given by adding to $M_{k}$ one of the contributions expressed in Eqs. (17) or (18).

\subsection{Model results}

We fit Eqs. (8) and (13) separately to the velocity $V\left(r_{N}\right)$ and velocity dispersion $\sigma\left(r_{N}\right)$ data points (see Sect. 5.2). The actual fit computation was done using the MPFIT algorithm, as done in Sect. 3.

One could add constraints to the halo parameter by including the disk $\mathrm{H} \beta$ rotation curve and the disk surface brightness profile into the fit. However, given the large uncertainties on the disk photometric profiles, we decided to focus our mass model only to the bulge regions. For completeness, we present the models in which the disk data are used in Appendix A.

The fit results are shown in Fig. 8 and are listed in Table 1. Fig. 8 shows the best fit model results (solid line) together with the observed radial profiles of velocity and velocity dispersion derived in Sect. 5.2. The last observed point of the rotation curve is below the best fit rotation curve, possibly the result of the bar influence on the stellar kinematics for $R \geq 3$ ". 5 as discussed in Sect. 5. Assuming this deviation is entirely due to the bar, a rough estimate of its effect on our results can be obtained from a fit of the velocity curve without taking into account the last velocity measurement. This is shown as the gray line in the upper panel of Fig. 8. The maximum difference between the two rotation curves (within $4 . .5$ ) is $\approx 14 \%$. Mass scales as the square of

\footnotetext{
${ }^{9}$ In Sect. 5 we assumed that the disk contribution to the bulge mass and dynamics is neglegible.
}
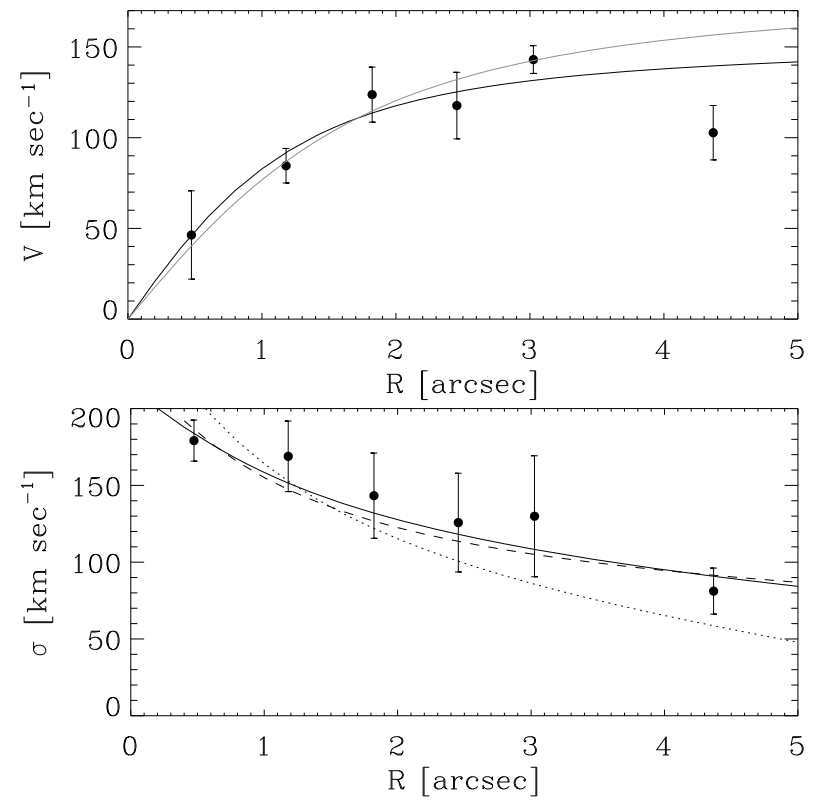

Fig. 8. Upper panel: stellar measured velocity curve of the bulge (filled circles, see Sect. 5.2) and the best fit model from Eq. (13). The black line represents the best rotation curve model, while the gray line represents the best rotation curve model excluding the last data point from the fit. Lower panel: measured radial velocity dispersion (filled circles, see Sect. 5.2) compared to the NFW (solid line), the pseudo isothermal (dashed line) and the self consistent (dotted line) best fit models.

Table 1. Bulge parameters from the best fit model. Values are converted assuming $D=194 \mathrm{Mpc}$ on the right column.

\begin{tabular}{lcc}
\hline \hline Parameter & Value & \\
\hline Rotation curve: & & \\
$v_{\infty}$ & $174 \pm 26\left[\mathrm{~km} \mathrm{~s}^{-1}\right]$ & \\
$r_{h}$ & $1.6 \pm 0.8[\operatorname{arcsec}]$ & $(1.5 \pm 0.8[\mathrm{kpc}])$ \\
\hline Self consistent & $\tilde{\chi}^{2}=3.6$ & \\
$M_{\mathrm{L}}$ & $6.6_{-1.4}^{+1.9}\left[10^{10} M_{\odot}\right]$ & \\
NFW: & $\tilde{\chi}^{2}=0.8$ \\
$M_{\mathrm{L}}$ & $2.3_{-2.5}^{3.5}+\left[10^{10} M_{\odot}\right]$ & \\
$\log _{10} \rho_{\mathrm{s}}$ & $10.1_{-0.2}^{+0.2}\left[\log _{10}\left(M_{\odot} / “ 3\right.\right.$ \\
$r_{\mathrm{S}}$ & $0.7_{-0.2}^{+0.2}[\operatorname{arcsec}]$ & $\left(\rho_{\mathrm{s}}=15_{-5}^{+9}\left[M_{\odot} \mathrm{pc}{ }^{-3}\right]\right)$ \\
Isothermal: & $\tilde{\chi}^{2}=1.5$ & $(0.67 \pm 0.19[\mathrm{kpc}])$ \\
$M_{\mathrm{L}}$ & $4.5_{-2}^{+3}\left[10^{10} M_{\odot}\right]$ & \\
$\log _{10} \rho_{0}$ & $9.6_{-0.4}^{+0.2}\left[\log _{10}\left(M_{\odot} /{ }^{\star 3}\right)\right]$ & $\left(\rho_{0}=5_{-1}^{+2}\left[M_{\odot} \mathrm{pc}{ }^{-3}\right]\right)$ \\
$r_{0}$ & $0.4_{-0.1}^{+0.1}[\operatorname{arcsec}]$ & $(0.38 \pm 0.09[\mathrm{kpc}])$ \\
\hline
\end{tabular}

the velocity, therefore we would expect a maximal underestimation of the total bulge mass of $\Delta M / M=2 \Delta V / V=28 \%$, which less than a factor of 1.3.

From Fig. 8 we can see that the self consistent model provides a poor fit to the data, compared to the dark matter scenarios. The bulge kinematics are therefore better explained, under our assumptions, with the presence of DM, although we are not able to disentangle between NFW and pseudo isothermal models. 

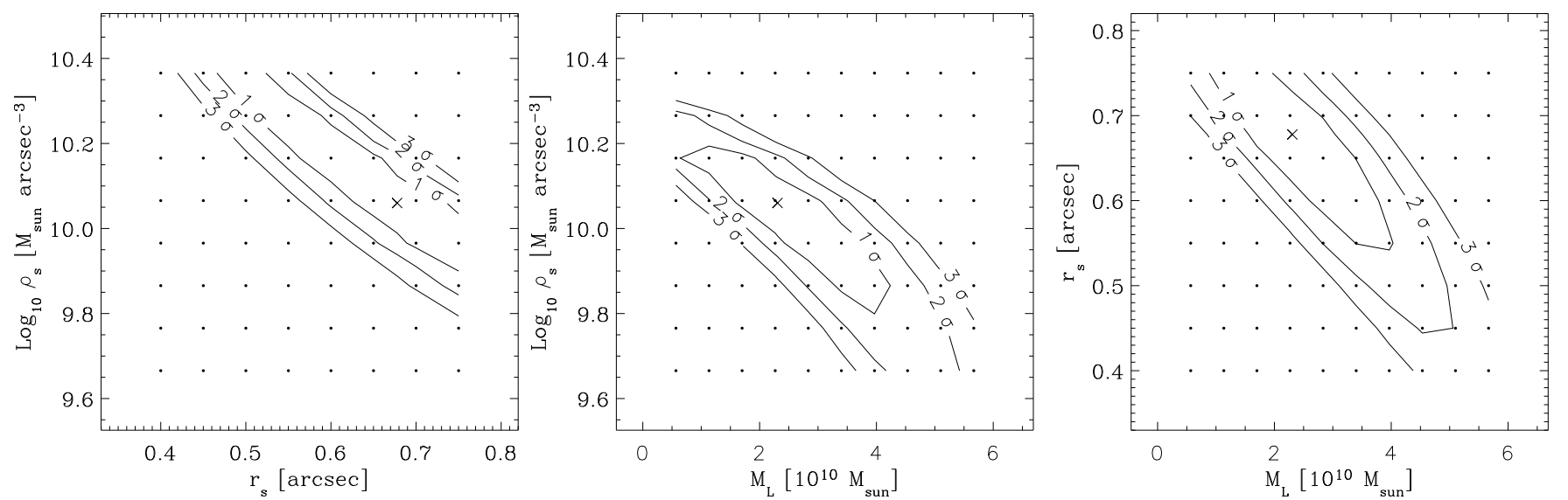

Fig. 9. $\chi^{2}$ confidence levels for the NFW dark matter halo model. The crosses represent the location of the best fit in the parameter space $\left(\tilde{\chi}^{2}=0.8\right)$.
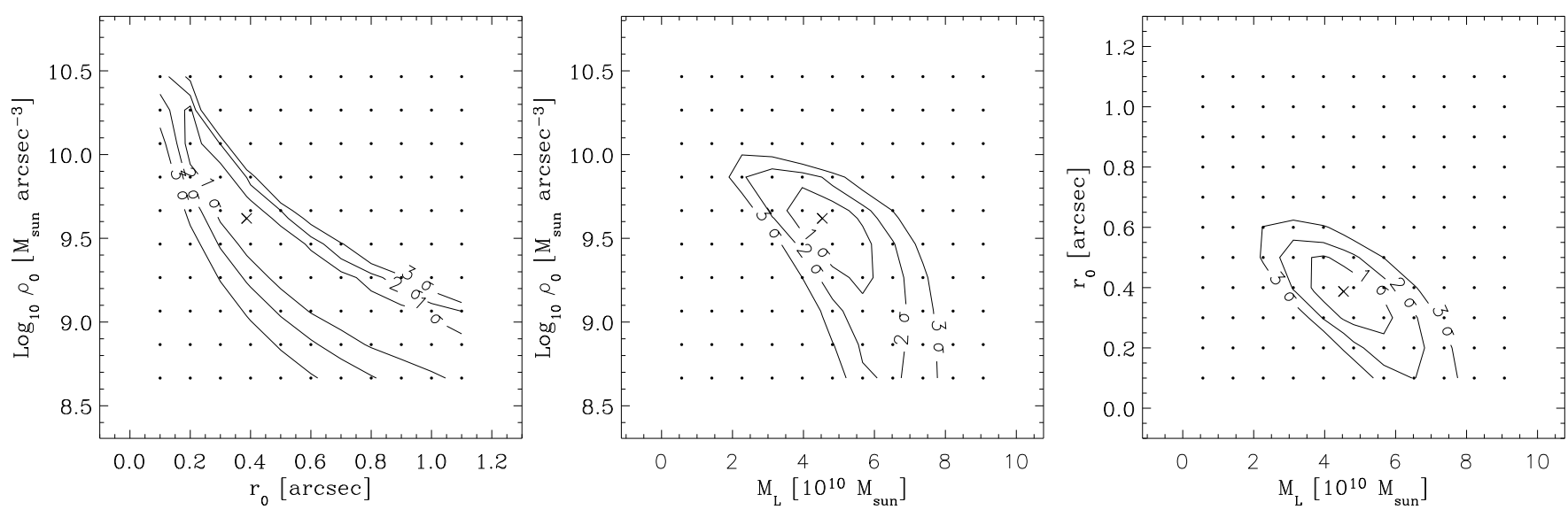

Fig. 10. $\chi^{2}$ confidence levels for the pseudo isothermal dark matter halo model. The crosses represent the location of the best fit in the parameter space $\left(\tilde{\chi}^{2}=1.5\right)$.

The reduced $\chi^{2}$ of both dark matter models is close to 1 , while the self consistent case gives a value of 3.6. In Figs. 9 and 10 we show the $1 \sigma, 2 \sigma$ and $3 \sigma \chi^{2}$ confidence levels in the parameter space. Those plots were produced by scaling the measured $\chi^{2}$ value to the ideal one $\chi^{2}=N-M=3(N=6$ is the number of data points, $M=3$ is the number of free parameters) and taking the expected $\Delta \chi^{2}$ variations for 3 parameters (i.e. 3.53, 8.02 and 14.2; Press et al. 1992, Chap. 15.6).

The total mass of the bulge (calculated using Eqs. (17) and (18) for $\left.r=5^{\prime \prime}\right)$ is $(7.4 \pm 3.2) \times 10^{10} M_{\odot}$ and $(7.1 \pm 3.6) \times$ $10^{10} M_{\odot}$ according to the NWF and pseudo isothermal scenarios, respectively, while the ratio of the dark matter content to the total mass is about 0.55 and 0.42 , at that radius. In the no dark matter scenario the bulge mass is $(6.5 \pm 1.6) \times 10^{10} M_{\odot}$. The total fraction of dark matter in the bulge, as a function of the radius, is shown in Fig. 11.

Errors of the bulge mass are computed by applying error propagation formulas to Eqs. (14), (17) and (18).

\subsubsection{Mass density radial profile}

From the measured stellar rotation curve and the radial velocity dispersion profiles (i.e. data points in Fig. 8) we derived the mass density profiles and compared them with the best model predictions.
First we calculated the circular velocity $V_{\mathrm{C}}$ using the asymmetric drift correction (assuming isotropy):

$V_{\mathrm{C}}^{2}\left(r_{N}\right)-v^{2}\left(r_{N}\right)=-\sigma^{2}\left(r_{N}\right) \frac{\partial \ln (v)}{\partial \ln \left(r_{N}\right)}$.

If we insert the observed bulge $r^{1 / 4}$ radial profile for the light distribution $v$ of the kinematic tracer we obtain:

$V_{\mathrm{C}}^{2}\left(r_{N}\right)=v^{2}\left(r_{N}\right)+1.92 \sigma^{2}\left(r_{N}\right)\left(\frac{r_{N}}{R_{\mathrm{e}}}\right)^{1 / 4}$.

Stellar mean circular velocity obtained with the asymmetric drift correction is $V_{\mathrm{c}}=243 \pm 6 \mathrm{~km} \mathrm{~s}^{-1}$ which is consistent with the one measured from the gas in the disk regions $\left(248 \pm 6 \mathrm{~km} \mathrm{~s}^{-1}\right)$.

Finally we calculated the mass density as done in de Blok et al. (2001) assuming a spherical mass distribution:

$\rho\left(r_{N}\right)=\frac{1}{4 \pi G}\left[\frac{V_{\mathrm{C}}^{2}}{r_{N}^{2}}+2 \frac{V_{\mathrm{C}}}{r_{N}}\left(\frac{\partial V_{\mathrm{C}}}{\partial R}\right)_{R=r_{N}}\right]$.

We did not fit these calculated mass density values because they do not contain more information than the observed $V$ and $\sigma$ themselves. Moreover, our direct fit to the observed quantities does not depend on the assumption adopted in the mass density calculation. We show in Fig. 12 the comparison between the derived $\rho$ and the prediction by the NFW and pseudo isothermal dark matter halo models. 


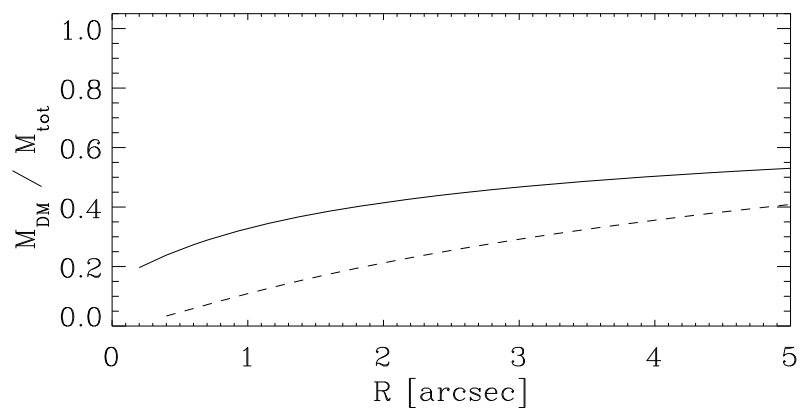

Fig. 11. Fraction of dark matter mass compared to total mass, as a function of radial distance, for the inner 5" of ESO 323-G064. NFW (solid line); pseudo isothermal (dashed line).

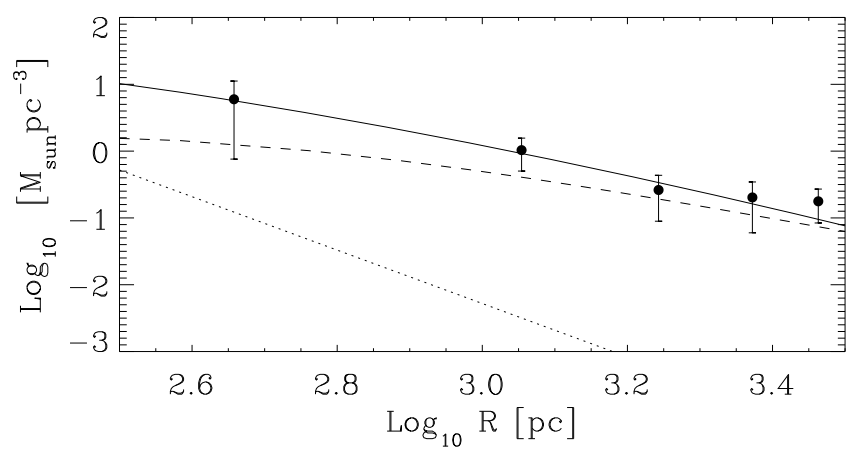

Fig. 12. Filled circles: mass density values derived from the observed velocity and velocity dispersion (Eq. (21)) compared to the best fit NFW (continuous line), pseudo isothermal (dashed line) and self consistent (dotted line) model predictions.

\section{Discussion and conclusions}

We presented the two-dimensional velocity and velocity dispersion fields for the gaseous and stellar components of the LSB galaxy ESO 323-G064. The gas emission lines show a very bright and complex structure within the central 5", characterized by 3 peaks, which we interpret as due to the presence of 3 spatially unresolved emitting regions. At radii out to $30^{\prime \prime}$, in the region dominated by the galaxy disk, a weak $\mathrm{H} \beta$ emission is detected showing a regular velocity field with a maximal amplitude of $248 \pm 6 \mathrm{~km} \mathrm{~s}^{-1}$.

The stellar absorption lines are detectable only in the very innermost regions of the bulge. The stellar kinematics shows a regular rotation with an amplitude of $\pm 140 \mathrm{~km} \mathrm{~s}^{-1}$ and a central velocity dispersion of $\approx 180 \mathrm{~km} \mathrm{~s}^{-1}$. The intrinsic $(V / \sigma)_{\text {INTR }}$ ratio for the bulge is $0.56 \pm 0.02$, which is consistent with an isotropic rotator in the $0.1<\epsilon_{\mathrm{OBS}}<0.2$ observed ellipticity range, considering an inclination of 62 degres. These values of $V / \sigma$ and $\epsilon$ place the bulge of ESO 323-G064 among fast rotator bulges (Cappellari et al. 2006). The value of $V / \sigma$ is considerably small when compared to the average value determined from the sample of 6 bulge dominated LSB galaxies of Pizzella et al. 2008 $(\langle V / \sigma\rangle>1)$.

The circular velocity, $248 \pm 6 \mathrm{~km} \mathrm{~s}^{-1}$ measured from the gaseous disk, places ESO 323-G064 in good agreement with the location of LSB galaxies in the $V_{\mathrm{C}}-\sigma_{\mathrm{c}}$ plane (Courteau et al. $2007 \mathrm{a}, \mathrm{b})$. On the other hand, this value is lower then the value of $320 \mathrm{~km} \mathrm{~s}^{-1}$ predicted by Pizzella et al. (2005).

The intrinsic bulge ellipticity value for ESO 323-G064 $\left(\epsilon_{\mathrm{INTR}}=0.20 \pm 0.07\right)$ is consistent with the mean value of bulges of the high surface brightness disk galaxies $\left\langle\epsilon_{\mathrm{HSB}}\right\rangle=0.15$ (as determined by Méndez-Abreu et al. 2008) while it is lower than the average value for bulge dominated LSB galaxies $\left\langle\epsilon_{\mathrm{LSB}}\right\rangle=0.45$ (as found by Pizzella et al. 2008a in a small sample of 6 bulge dominated LSB).

The amplitude of the gaseous rotation curve $\left(248 \pm 6 \mathrm{~km} \mathrm{~s}^{-1}\right)$ leads to an estimate of the total baryonic mass in the galaxy of $M_{\text {bar }}=(1.9 \pm 0.2) \times 10^{11} M_{\odot}$ using the empirical baryonic Tully Fisher, as done in McGaugh (2005). Moreover, under the hypothesis of a $\Lambda$ CDM universe (see Sect. 3.1 for details on the assumed parameters), we estimate the total mass for the dark matter halo $M_{\mathrm{DM}} \sim 5 \times 10^{12} M_{\odot}$.

We produce spherical isotropic Jeans models for the stellar kinematics in the bulge, exploring the self consistent, NFW and pseudo isothermal scenarios. Even though the data are to be taken with some caveats (due to the lack of good photometry, limited spatial extension and resolution of the stellar kinematics) with this simple analysis we show that dark matter scenarios fit the data better than the self consistent model. The derived total bulge mass is $(7 \pm 3) \times 10^{10} M_{\odot}$ but we are not able to disentangle between the two different dark matter models.

The derived central bulge mass density (see Table 1) is $\rho=15_{-5}^{+9}\left[M_{\odot} \mathrm{pc}^{-3}\right]$ in the NFW scenario, and $\rho=5_{-1}^{+2}\left[M_{\odot} \mathrm{pc}^{-3}\right]$ in the pseudo isothermal scenario. Typical values of central mass density range from few $10^{-3} M_{\odot} \mathrm{pc}^{-3}$ to few $10^{-2} M_{\odot} \mathrm{pc}^{-3}$ for regular low surface brightness (see for example Kuzio de Naray et al. 2006; de Blok et al. 2001) and giant low surface brightness galaxies (Pickering et al. 1997). On the contrary, a much wider range of values is measured for regular galaxies, from few $10^{-3} M_{\odot} \mathrm{pc}^{-3}$ to several $10^{3} M_{\odot} \mathrm{pc}^{-3}$ (i.e. Salucci \& Borriello 2001; Noordermeer et al. 2007). Therefore, in this picture, the bulge of ESO 323-G064 resembles more the central mass density of regular bulges than those measured in low surface brightness galaxies. This is consistent also with the fact that bulges of giant LSB galaxies are photometrically similar to those of regular high surface brigthtness galaxies (McGaugh et al. 1995; Beijersbergen et al. 1999).

Acknowledgements. The authors wish to thank the referee A. Bosma for useful suggestions which improved the paper content and the discussion.

\section{Appendix A: Constraining the halo parameters with disk $\mathrm{H} \beta$ velocity curve and surface brightness}

The $\mathrm{H} \beta$ velocity curve measured in the disk between $10^{\prime \prime}<R<$ $30^{\prime \prime}$ (Sect. 3.1) and the disk surface photometry from the ESOLV images can be used to constrain the dark halo parameters, under the assumption that the measured $\mathrm{H} \beta$ velocity curve is a good representation of the galaxy circular velocity.

\section{A.1. Circular velocity for the disk and the halo}

The circular velocity predicted for a pseudo isothermal halo is:

$V_{\text {isot }}(r)=V_{\mathrm{H}} \sqrt{1-\frac{r_{0}}{r} \arctan \left(\frac{r}{r_{0}}\right)}$

where $\left.V_{\mathrm{H}}=\sqrt{\left(4 \pi G \rho_{0}\right.} r_{0}^{2}\right)$ and $\rho_{0}, r_{0}$ are the halo parameters defined in Sect. 5.3.

The circular velocity predicted for a NFW halo is (Navarro et al. 1997):

$V_{\mathrm{NFW}}(r)=V_{200}\left[\frac{1}{x} \times \frac{\ln (1+c x)-c x /(1+c x)}{\ln (1+c)+c /(1+c)}\right]^{1 / 2}$ 
Table 2. Galfit best fit parameters.

\begin{tabular}{lcc}
\hline \hline & $R$-band & $B$-band \\
\hline Bulge: & & \\
$M_{\mathrm{tot}}$ & $16.23 \pm 0.5 \mathrm{mag}$ & $17.75 \pm 0.5 \mathrm{mag}$ \\
$R_{\mathrm{e}}$ & $0.65 \pm 1 \mathrm{arcsec}$ & $0.55 \pm \mathrm{arcsec}$ \\
$b / a$ & $0.71 \pm 0.3$ & $0.71 \pm 0.3$ \\
$\mathrm{PA}$ & $13 \pm 20 \mathrm{deg}$ & $-72 \pm 20 \mathrm{deg}$ \\
\hline Disk: & & \\
$M_{\text {tot }}$ & $14.11 \pm 0.5 \mathrm{mag}$ & $15.81 \pm 0.5 \mathrm{mag}$ \\
$\mu_{0}$ & $21.2 \pm 0.4 \mathrm{mag}$ & $23.3 \pm 0.4 \mathrm{mag}$ \\
$h$ & $12.4 \pm 1 \mathrm{arcsec}$ & $13.6 \pm 2 \mathrm{arcsec}$ \\
$b / a^{(*)}$ & $0.53 \pm 0.3$ & $0.52 \pm 0.3$ \\
$\mathrm{PA}$ & $33 \pm 5 \mathrm{deg}$ & $32 \pm 6 \mathrm{deg}$ \\
\hline
\end{tabular}

Note: ${ }^{(*)}$ A disk axial ratio $b / a=0.53$ corresponds to an inclination $i=60^{\circ}$, assuming an intrinsic disk axial ratio of $q_{0}=0.18$ (Guthrie 1992).

In Eq. (23) $c$ is the concentration parameter, related to the density parameter $\rho_{\mathrm{s}}$ (used in our fit procedure) and the critical density $\rho_{\text {critic }}=3 H^{2} /(8 \pi G)(H=75$ is the adopted value for the Hubble constant) with the following equation:

$\rho_{\mathrm{s}} / \rho_{\text {critic }}=\frac{200}{3} \frac{c}{\ln (1+c)-c /(1+c)}$.

The parameter $V_{200}$ in Eq. (23) is the circular velocity at $r_{200}=$ $r_{\mathrm{s}} c\left(r_{\mathrm{s}}\right.$ is the halo scale parameter used in our fit procedure) and it is defined as:

$V_{200}=\left(\frac{G M_{200}}{r_{200}}\right)^{1 / 2}$

where

$M_{200}=200 \frac{4 \pi}{3} \times \rho_{\text {critic }} r_{200}^{3}$.

Together with the halo circular velocities defined by Eqs. (22) and (23) we have to add the contribution given by the stellar disk (the bulge contribution is negligible at this radial range). Assuming an exponential disk with central mass surface density $M_{0}$ and scale length $h$, the predicted circular velocity is (Freeman 1970):

$V_{\text {disk }}(r)=\sqrt{4 \pi G M_{0} \frac{r^{2}}{4 h}\left[I_{0}\left(\frac{x}{2 h}\right) K_{0}\left(\frac{x}{2 h}\right)-I_{1}\left(\frac{x}{2 h}\right) K_{1}\left(\frac{x}{2 h}\right)\right]}$

where $I_{n}$ and $K_{n}$ are modified Bessel functions of the first and second kind.

To obtain the mass surface density $M_{0}$ and the disk scale parameter we retrieved ESO-LV images of ESO 323-G064 in the $B$ and $R$ bands and performed a photometric decomposition with galfit (Peng et al. 2002).

The result of the galfit decomposition are listed in Table 2. From the total disk magnitude in $R$ and $B$ band we compute a color of $(B-R)_{\text {disk }}=1.70 \pm 0.5$. Using the prescription by Bell $\&$ de Jong (2001) we can compute the mass-to-light ratio of the disk in the $R$ band $(M / L)_{\text {disk }}=4.81 \pm 0.77$. The computation made use of the relation between color and mass-to-light ratio for different models as listed in Table 3 of Bell \& de Jong (2001). We use the relation in the $R$ because the scatter between different models is smaller.

From the central disk surface brightness $\mu_{0}=21.2 \pm$ 0.3 mag $\operatorname{arcsec}^{-2}$ measured from the galfit decomposition in the $R$-band, assuming a distance of $D=194 \mathrm{Mpc}$, an extinction of $A_{R}=0.231 \mathrm{mag}$ (from the NED database) the disk
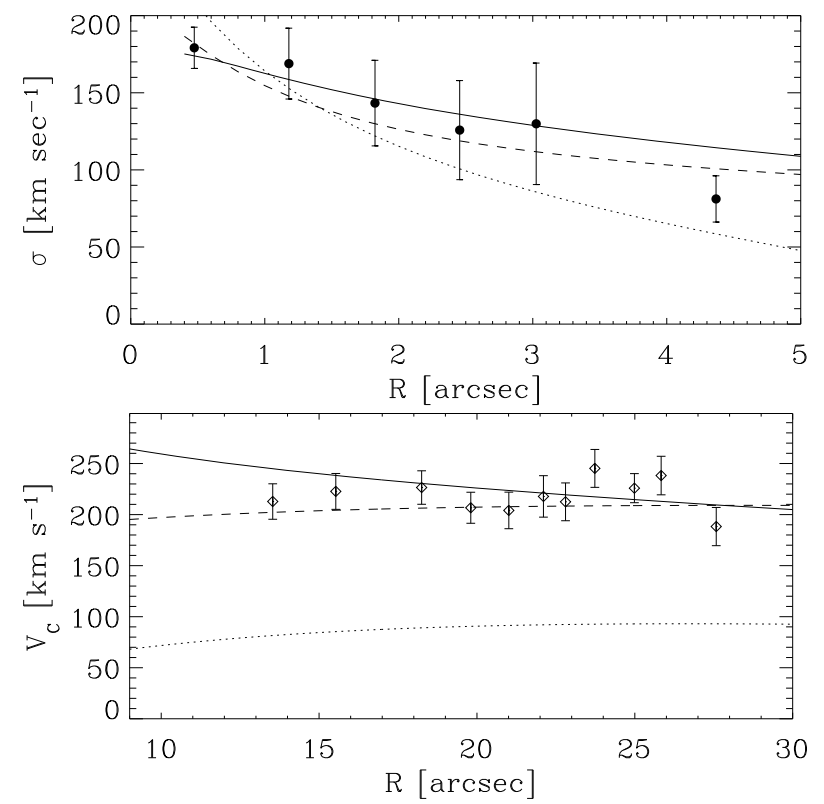

Fig. 13. Model results obtained including the disk data. Upper panel: stellar velocity dispersion (filled circles, see Sect. 5.2) compared to the NFW (solid line), the pseudo isothermal (dashed line) and the self consistent (dotted line) best fit models. Lower Panel: measured circular velocity (open circles, from the $\mathrm{H} \beta$ rotation curve derived in Sect. 3.1) compared to the NFW (solid line), the pseudo isothermal (dashed line) and the self consistent (dotted line) best fit models.

mass-to-light ratio $(M / L)_{\text {disk }}=4.81 \pm 0.77$ we calculate the central mass surface density $M_{0}=(491 \pm 201) M_{\odot} \mathrm{pc}^{-2}=$ $(4.3 \pm 1.8) \times 10^{8} M_{\odot} \operatorname{arcsec}^{-2}$.

Therefore, we can compute the total circular velocity $V_{C}$ by adding the contribution of the light (Eq. (27)) to the contribution of the halo (Eqs. (22) or (23) depending on the adopted scenario).

\section{A.2. Mass model using stellar and gaseous kinematics}

We have also performed the mass model fit as described in Sect. 5 using the constraints from the $\mathrm{H} \beta$ circular velocity. As in the previous case we fit first the empirical bulge stellar rotation curve (to obtain $r_{\infty}$ and $r_{h}$, Eq. (12)) and then we fit simultaneously the stellar velocity dispersion (i.e the filled circles in Fig. 8) and the $\mathrm{H} \beta$ circular velocity (i.e the open diamonds in Fig. 2) data.

Fit results are shown in Fig. 13 and the best fit parameters are listed in Table 3. The results are similar to those in Table 1. With the additional constraints from the disk, both pseudo isothermal and NFW scenario give a reasonable fit to the data and it is not possible, given the errors, to say which is the best one.

The formal errors given by the fit algorithm are slightly lower than the ones listed in Table 1 , because of the addition of data points in the fit. However, the fit errors do not include the uncertainties from the disk photometry and the errors in the relation between mass-to-light ratio and disk $(B-R)$ color.

Errors in disk central surface brightness and in the mass-tolight ratio translate into an error of $\Delta M_{0} / M_{0} \sim 41 \%$ on the mass central surface density, which is an error of $\sim 20 \%$ on the disk velocity curve $\left(V_{\text {disk }} \propto \sqrt{M_{0}}\right)$. However, the fact that the two different fit approaches lead to similar results is reassuring. 
Table 3. Best fit parameters from the simultaneous fit of the stellar velocity dispersion and the $\mathrm{H} \beta$ rotation curve. Values are converted assuming $D=194 \mathrm{Mpc}$ on the right column.

\begin{tabular}{lcc}
\hline \hline Parameter & Value & \\
\hline Rotation curve: & & \\
$v_{\infty}$ & $174 \pm 26\left[\mathrm{~km} \mathrm{~s}^{-1}\right]$ & \\
$r_{\mathrm{h}}$ & $1.6 \pm 0.8[\operatorname{arcsec}]$ & $(1.5 \pm 0.8[\mathrm{kpc}])$ \\
\hline Self consistent & $\tilde{\chi}^{2}=3.6$ & \\
$M_{\mathrm{L}}$ & $6.6_{-1.4}^{+1.9}\left[10^{10} M_{\odot}\right]$ & \\
NFW: & $\tilde{\chi}^{2}=0.9$ \\
$M_{\mathrm{L}}$ & $0.54_{-0.4}^{1.0}+\left[10^{10} M_{\odot}\right]$ & \\
$\log _{10} \rho_{\mathrm{s}}$ & $10.0_{-0.2}^{+0.2}\left[\log _{10}\left(M_{\odot} /{ }^{\star 33}\right)\right]$ & $\left(\rho_{\mathrm{s}}=13_{-5}^{+8}\left[M_{\odot} \mathrm{pc}{ }^{-3}\right]\right)$ \\
$r_{\mathrm{S}}$ & $0.85_{-0.15}^{+0.14}[\operatorname{arcsec}]$ & $(0.80 \pm 0.18[\mathrm{kpc}])$ \\
Isothermal: & $\tilde{\chi}^{2}=1.4$ & \\
$M_{\mathrm{L}}$ & $3.5_{-2}^{+3}\left[10^{10} M_{\odot}\right]$ & \\
$\log _{10} \rho_{0}$ & $9.6_{-0.4}^{+0.2}\left[\log _{10}\left(M_{\odot} /{ }^{\star 3}\right)\right]$ & $\left(\rho_{0}=5_{-1}^{+2}\left[M_{\odot} \mathrm{pc}{ }^{-3}\right]\right)$ \\
$r_{0}$ & $0.39_{-0.09}^{+0.08}[\operatorname{arcsec}]$ & $(0.37 \pm 0.08[\mathrm{kpc}])$ \\
\hline
\end{tabular}

Deep photometric observations and an accurate determination of the mass-to-light ratio are highly desirable to better constrain the mass distribution in ESO 323-G064.

\section{References}

Beijersbergen, M., de Blok, W. J. G., \& van der Hulst, J. M. 1999, A\&A, 351, 903

Bell, E. F., \& de Jong, R. S. 2001, ApJ, 550, 212

Binney, J. 1978, MNRAS, 183, 501

Binney, J. 2005, MNRAS, 363, 937

Binney, J., \& Tremaine, S. 1987, Galactic dynamics (Princeton, NJ: Princeton University Press), 747

Boissier, S., Gil de Paz, A., Boselli, A., et al. 2008, ArXiv e-prints, 803

Bothun, G., Impey, C., \& McGaugh, S. 1997, PASP, 109, 745

Bothun, G. D., Schombert, J. M., Impey, C. D., \& Schneider, S. E. 1990, ApJ, 360,427

Bryan, G. L., \& Norman, M. L. 1998, ApJ, 495, 80

Bullock, J. S., Kolatt, T. S., Sigad, Y., et al. 2001, MNRAS, 321, 559

Cappellari, M., \& Emsellem, E. 2004, PASP, 116, 138

Cappellari, M., Bacon, R., Bureau, M., et al. 2006, MNRAS, 366, 1126

Cappellari, M., Emsellem, E., Bacon, R., et al. 2007, MNRAS, 379, 418
Courteau, S., McDonald, M., \& Widrow, L. M. 2007a, [arXiv:0709. 3682]

Courteau, S., McDonald, M., Widrow, L. M., \& Holtzman, J. 2007b, ApJ, 655, L21

de Blok, W. J. G., \& McGaugh, S. S. 1997, MNRAS, 290, 533

de Blok, W. J. G., van der Hulst, J. M., \& Bothun, G. D. 1995, MNRAS, 274, 235

de Blok, W. J. G., McGaugh, S. S., Bosma, A., \& Rubin, V. C. 2001, ApJ, 552, L23

Freeman, K. C. 1970, ApJ, 160, 811

Guthrie, B. N. G. 1992, A\&AS, 93, 255

Hernquist, L. 1990, ApJ, 356, 359

Hoffman, Y., Silk, J., \& Wyse, R. F. G. 1992, ApJ, 388, L13

Hui, X., Ford, H. C., Freeman, K. C., \& Dopita, M. A. 1995, ApJ, 449, 592

Jimenez, R., Bowen, D. V., \& Matteucci, F. 1999, ApJ, 514, L83

Kuzio de Naray, R., McGaugh, S. S., de Blok, W. J. G., \& Bosma, A. 2006, ApJS, 165, 461

Kuzio de Naray, R., McGaugh, S. S., \& de Blok, W. J. G. 2008, ApJ, 676, 920

Lauberts, A. 1982, ESO/Uppsala survey of the ESO(B) atlas (Garching: European Southern Observatory (ESO))

Mapelli, M., Moore, B., Ripamonti, E., et al. 2008, MNRAS, 383, 1223

Mayer, L., \& Wadsley, J. 2004, MNRAS, 347, 277

McGaugh, S. S. 2005, ApJ, 632, 859

McGaugh, S. S., Schombert, J. M., \& Bothun, G. D. 1995, AJ, 109, 2019

McGaugh, S. S., Rubin, V. C., \& de Blok, W. J. G. 2001, AJ, 122, 2381

Méndez-Abreu, J., Aguerri, J. A. L., Corsini, E. M., \& Simonneau, E. 2008, A\&A, 478, 353

Moré, J. J., Garbow, B. S., \& Hillstrom, K. E. 1980, User Guide for MINPACK-1 (Argonne Nat. Lab. Rep. ANL-80-74; Argonne: Argonne National Laboratory)

Navarro, J. F., Frenk, C. S., \& White, S. D. M. 1997, ApJ, 490, 493

Noguchi, M. 2001, MNRAS, 328, 353

Noordermeer, E., van der Hulst, J. M., Sancisi, R., Swaters, R. S., \& van Albada, T. S. 2007, MNRAS, 376, 1513

O’Neil, K. 2002, in Extragalactic Gas at Low Redshift, ed. J. S. Mulchaey, \& J. Stocke, ASP Conf. Ser., 254, 202

O’Neil, K., \& Schinnerer, E. 2003, ApJ, 588, L81

Osterbrock, D. E., Fulbright, J. P., Martel, A. R., et al. 1996, PASP, 108, 277

Peng, C. Y., Ho, L. C., Impey, C. D., \& Rix, H.-W. 2002, AJ, 124, 266

Peng, E. W., Ford, H. C., \& Freeman, K. C. 2004, ApJ, 602, 685

Pfenniger, D., Combes, F., \& Martinet, L. 1994, A\&A, 285, 79

Pickering, T. E., Impey, C. D., van Gorkom, J. H., \& Bothun, G. D. 1997, AJ, 114, 1858

Pizzella, A., Corsini, E. M., Dalla Bontà, E., et al. 2005, ApJ, 631, 785

Pizzella, A., Corsini, E. M., Sarzi, M., et al. 2008a, MNRAS, 387, 1099

Pizzella, A., Tamburro, D., Corsini, E. M., \& Bertola, F. 2008b, A\&A, 482, 53

Press, W. H., Teukolsky, S. A., Vetterling, W. T., \& Flannery, B. P. 1992, Numerical recipes in FORTRAN. The art of scientific computing (Cambridge: University Press), 2nd edn.

Salucci, P., \& Borriello, A. 2001, in Dark Matter in Astro- and Particle Physics, ed. H. V. Klapdor-Kleingrothaus, 12

Sánchez-Blázquez, P., Peletier, R. F., Jiménez-Vicente, J., et al. 2006, MNRAS, 371,703

Swaters, R. A., Madore, B. F., \& Trewhella, M. 2000, ApJ, 531, L107

Swaters, R. A., Verheijen, M. A. W., Bershady, M. A., \& Andersen, D. R. 2003, ApJ, 587, L19

Valdes, F., Gupta, R., Rose, J. A., Singh, H. P., \& Bell, D. J. 2004, ApJS, 152, 251 